February 28, $2022 \quad$ 22:26 WSPC/INSTRUCTION FILE ele ijmpd’arxiv

International Journal of Modern Physics D

(C) World Scientific Publishing Company

\title{
The electron plus positron spectrum from annihilation of Kaluza-Klein dark matter in the Galaxy
}

\author{
Satoshi Tsuchida* \\ Department of Physics, Osaka City University, 3-3-138 Sugimoto, Sumiyoshi-ku, Osaka City, \\ Osaka, 558-8585, Japan \\ stsuchida88@gmail.com \\ Masaki Mori \\ Department of Physical Sciences, Ritsumeikan University, Kusatsu 525-8577, Shiga, Japan \\ Received Day Month Year \\ Revised Day Month Year
}

\begin{abstract}
The lightest Kaluza-Klein particle (LKP), which appears in the theory of universal extra dimensions, is one of good candidates for cold dark matter (CDM). When LKP pairs annihilate around the center of the Galaxy where CDM is concentrated, there are some modes which produce electrons and positrons as final products, and we categorize them into two components. One of them is the "Line" component, which directly annihilates into electron-positron pair. Another one is the "Continuum" component, which consists of secondarily produced electrons and positrons via some decay modes. Before reaching Earth, directions of electrons and positrons are randomized by the Galactic magnetic field, and their energies are reduced by energy loss mechanisms. We assume the LKP is in the mass range from $300 \mathrm{GeV}$ to $1500 \mathrm{GeV}$. We calculate the electron plus positron spectrum after propagation in the Galactic halo to Earth, and we analyze the resulting spectrum and positron fraction. We also point out that the energy dependence of observed positron fraction is well reproduced by the mixture of "line" and "continuum" components. We can fit the electron plus positron spectrum and the positron fraction by assuming appropriate boost factors describing dark matter concentration in the Galactic halo. However, it is difficult to explain both the electron plus positron spectrum and the positron fraction by a single boost factor, if we take account of observational data obtained by AMS-02 only.
\end{abstract}

Keywords: Dark matter; Kaluza-Klein particle; Cosmic-ray electron; Cosmic-ray positron.

PACS numbers: 95.35.+d; 98.70.Sa

\section{Introduction}

The fact that most of the matter in the Universe consists of non-baryonic dark matter ${ }^{[1}$ is supported further by the Planck observational data, ${ }^{2}$ and dark matter should be made of particles which do not exist in the standard model of particle physics. Recent observations of cosmic positron excess ${ }^{3}{ }^{3}$ could be explained by secondarily produced positrons in annihilation of dark matter particles in the Galactic halo, as 
is discussed by many authors (see, e.g. Refs $\underline{6}$ ( $)$ ). Among various candidates of dark matter, the lightest Kaluza-Klein particle (LKP), predicted in the theory of universal extra dimensions (UED), 911 is unique since there would be a characteristic edge structure in the cosmic electron plus positron spectrum near the LKP mass as Cheng et al $!^{12}$ predicted. The edge structure was calculated by Moiseev et al $!^{13}$ for Fermi-LAT detection, but, at least in the energy range below $1000 \mathrm{GeV}$, such structure has not established so far (see, e.g. Refs. $\frac{414}{15}$ ). On the other hand, above 1000 $\mathrm{GeV}$, the observational data are still limited, so the characteristic structure could be observed in near-future missions. For example, the Calorimetric Electron Telescope (CALET), which is a Japanese-led detector and is a fine resolution calorimeter for cosmic-ray observation installed on the International Space Station in August 2015, started exploring the energy range up to $20 \mathrm{TeV}$ for electrons and positrons. 16

In this paper, we calculate the electron and positron spectrum and the positron fraction from LKP annihilation in the Galactic halo including the effects of propagation, and compare the results with recent measurements. We found the energy dependence of the positron fraction is well explained by the mixture of "line", which consists of electron-positron pairs directly produced by annihilation, and "continuum" component, which consists of secondarily produced electrons and positrons. Also the total electron plus positron spectrum can be explained by taking account of the LKP contribution assuming appropriate values for the boost factor describing concentration of dark matter in the Galactic halo. Then we discuss the constraints on the boost factor.

\section{Production of electrons and positrons}

In UED assuming only one extra dimension, the extra dimension is compactified with radius $R$, and the LKP mass, which we denote as $m_{B^{(1)}}$, is inversely proportional to $R$. The relevant mass for the LKP ranges from a few $100 \mathrm{GeV}$ to $1000 \mathrm{GeV}, 17$ if we assume the LKP contributes significantly to cold dark matter. More recently, progress in estimating the relic density indicate it could be as heavy as $1.5 \mathrm{TeV}{ }^{18}$ Experimentally, recent LHC results indicate the LKP mass lighter than $950 \mathrm{GeV}$ is disfavored ${ }^{19}$ Here, we vary the LKP mass from $300 \mathrm{GeV}$ to 1500 $\mathrm{GeV}$, and we analyze the electron plus positron spectrum and the positron fraction to be observed at Earth from LKP annihilation.

When LKP pairs annihilate, there are some modes which produce electrons and positrons as final products, and we categorize them into two components. One of them is a "line" component, which consists of electron-positron pairs directly produced by annihilation, and gives rise to edge structure near the LKP mass after propagating in the Galactic halo to Earth. Another is a "continuum" component, which consists of secondarily produced electrons and positrons via muon pairs, tauon pairs, quark pairs, and gauge bosons produced by LKP annihilation. The branching ratios are given as follows: $20 \%$ for charged leptons, $11 \%$ for up-type quarks, $0.7 \%$ for down-type quarks, $1 \%$ for charged gauge bosons, and $0.5 \%$ for neutral gauge 


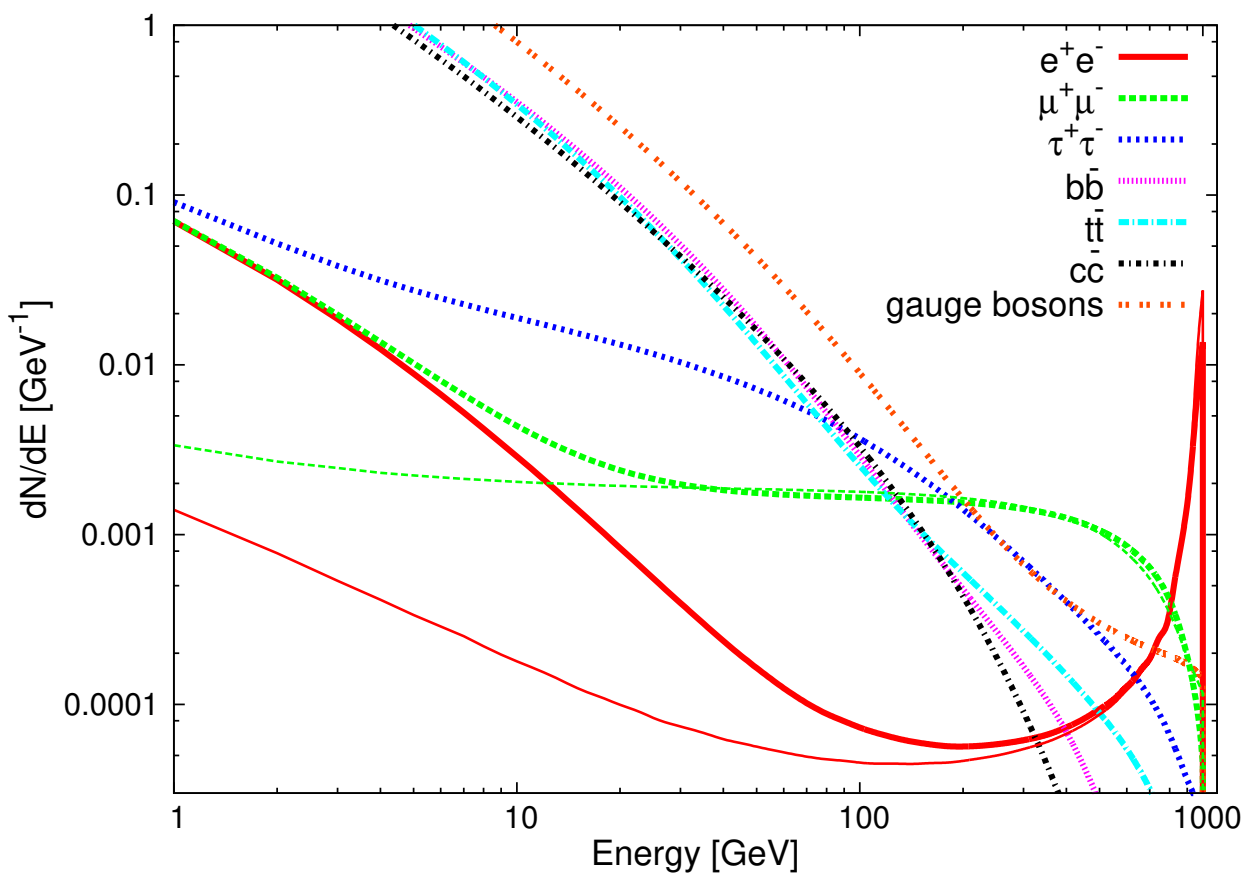

Fig. 1. (Color Online). The "continuum" and "line" positron spectra from LKP annihilations for $m_{B^{(1)}}=1000 \mathrm{GeV} 2122 \mathrm{The}$ patterned lines correspond to the positron spectra per annihilation via muon pairs, tauon pairs, quark pairs, and gauge bosons, respectively. The solid line corresponds to the line component. The thick lines include the electroweak corrections, and the thin lines do not include those corrections.

bosons, respectively $\frac{11,20}{20}$ We the spectra for line and continuum components given by Cirelli et al! 21 and Ciafaloni et al. $\stackrel{221}{2}$ which are shown in Fig. 11 assuming $100 \%$ branching ratios for each component, where the solid line indicates the line spectrum and patterned lines show the continuum spectra from muon pairs, tauon pairs, quark pairs $(b, t, c)$, and gauge bosons, respectively. Note that the line spectra shows a tail toward lower energies due to final state interactions. For comparison, the positron spectra without electroweak corrections are shown in thin lines for the line spectrum and the continuum spectrum for muon pairs. One can see the electroweak correction affects the spectra in the lower energy region.21]22]

The spectra for line and continuum components shown in Fig. 1 are those just after pair annihilation, and we have to take account of the effects of propagation in the Galactic halo to Earth, such as diffusion and energy loss processes. For this purpose, we follow the Green function approach given by Moskalenko and Strong, 23 assuming the "Isothermal model" as the halo profile for reference. In addition, we should include a "boost factor", $B_{f}$, which describes the signal enhancement from dark matter annihilation in the Galactic halo. ${ }^{24} N$-body simulation study given by Navarro, Frenk and White (NFW) $\stackrel{25}{2}$ for example, indicates a large $B_{f}$. The boost 
factor $B_{f}$ is defined as following expression

$$
\begin{aligned}
B_{f} & =B_{\rho} \times B_{\sigma v} \\
& =\left(\frac{\left\langle\rho^{2}(l)\right\rangle_{\Delta V}}{\left\langle\rho_{0}^{2}(l)\right\rangle_{\Delta V}}\right)\left(\frac{\langle\sigma v\rangle}{3 \times 10^{-26} \mathrm{~cm}^{3} \mathrm{~s}^{-1}}\right)_{\Delta V}
\end{aligned}
$$

where $\rho_{0}$ is a local dark matter density estimated as $0.43 \mathrm{GeV} / \mathrm{cm}^{3}$ for "Isothermal" halo model, $\frac{23}{23}$ and $3 \times 10^{-26} \mathrm{~cm}^{3} \mathrm{~s}^{-1}$ is the typical cross section for cold dark matter annihilation.26

The values of $B_{f}$ could be determined to fit the observed positron fraction as discussed extensively to interpret the "anomaly" reported by PAMELA. For example, Cirelli et al. discussed the values of $B_{f}$ for each annihilation mode.27 However, the energy dependence of the positron fraction observed by AMS-02 with more statistics 5 is not well explained if only the line (or continuum) component is taken into account. We will show later that we can fit it well by considering both the line and the continuum components assuming an appropriate boost factor $B_{f}$.

\section{The effect of propagation}

Charged particles, such as electrons and positrons, produced by LKP annihilation around the center of the Galaxy change their direction randomly by the irregular component in the Galactic magnetic field, and lose their energies by bremsstrahlung in interstellar matter before reaching Earth. Thus, the observational electron plus positron fluxes have different shapes from initial ones. The effects of propagation are studied by Moskalenko and Strong,23 and we calculate the modulated flux by using their results given as Green functions.

The positron flux is given by,

$$
\frac{d \Phi_{e^{+}}}{d \Omega d E}=\langle\sigma v\rangle B_{f}\left(\frac{\rho_{0}}{m_{B^{(1)}}}\right)^{2} \sum_{i} B_{i} \int d \epsilon \frac{d N_{i}}{d \epsilon} g(\epsilon, E) \mathrm{cm}^{-2} \mathrm{~s}^{-1} \mathrm{sr}^{-1} \mathrm{GeV}^{-1}
$$

where $B_{i}$ is a branching ratio for each particle, and $\rho_{0}$ is the local dark matter density. The annihilation cross section $\langle\sigma v\rangle$ to yield the significant relic density of cold dark matter is the order of $3 \times 10^{-26} \mathrm{~cm}^{3} \mathrm{~s}^{-1} \underline{26}$

The Green function, $g(\epsilon, E)$, is defined as

$$
g(\epsilon, E)=\frac{10^{25}}{E^{2}} 10^{a\left(\log _{10} E\right)^{2}+b\left(\log _{10} E\right)+c} \theta(\epsilon-E)
$$

where $E$ is the observed energy in $\mathrm{GeV}$ and the parameters $a, b$ and $c$ are tabulated in Ref., ${ }^{23}$ which gives the spectra of electron and positron after propagation, for monochromatic energy $(\epsilon)$ injection.

In addition, we should take account of the effects of solar modulation in the low energy region below $10 \mathrm{GeV}$. The magnetic field of the Sun is the source of the observed modulation of the Galactic cosmic rays. Solar modulation is dominant on low energy particles, and affects on spectral shape for cosmic rays. In the force field 


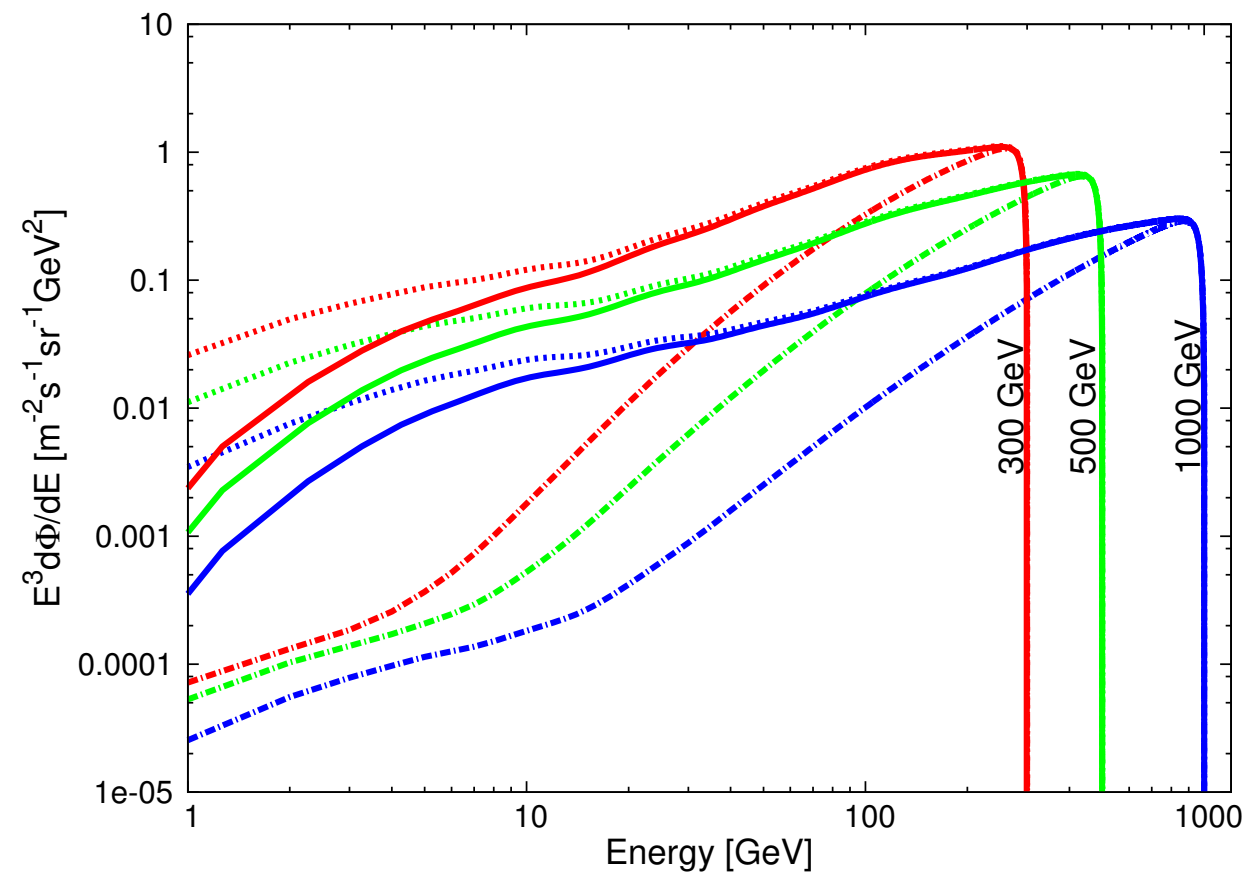

Fig. 2. (Color Online). The spectra of electrons plus positrons from LKP annihilation after propagation for three assumed LKP masses. The dot-dashed lines show the spectrum without solar modulation for the "line" component only, the dotted lines show the total spectrum from LKP annihilation ("continuum" plus "line") without solar modulation, and the solid lines show the total spectrum including effect of solar modulation with the solar modulation potential $\phi=735 \mathrm{MV}$. We assume the boost factor $B_{f}=1$.

approximation, the differential flux of particles of mass $m$ and charge $Z e, \Phi(E)$, reaching Earth with energy $E$ is related to the interstellar flux, $\Phi\left(E_{I S}\right)$, as

$$
\Phi(E)=\frac{E^{2}-m^{2}}{E_{I S}^{2}-m^{2}} \Phi\left(E_{I S}\right)
$$

where $E_{I S}$ is the energy in interstellar space and related to $E$ as $E=E_{I S}-|Z| \phi$, and $\phi$ is a solar modulation potential..$^{28}$

The line component is approximately in the form of $\delta$-function before propagation in the Galactic halo. However, its spectrum after propagation in the Galactic halo to Earth extends to lower energies caused by the effects of diffusion and energy loss processes. In addition to the line component, we also calculate the continuum component. The continuum component has a broad spectrum extending to lower energies when it is produced by LKP annihilation as shown in Fig. 1. Then we calculated the spectrum after propagation using the Green function similarly to the case of the line component. These results for the case of the isothermal halo density profile are shown in Fig. 2. where the dot-dashed lines show the spectrum for the 
line component only without solar modulation, the dotted lines show the total spectrum from LKP annihilation (continuum plus line) without solar modulation, and solid lines show the total spectrum including effect of solar modulation assuming a solar modulation potential $\phi=735 \mathrm{MV}^{29}$ for three assumed LKP masses. This figure indicates the continuum component becomes dominant in lower energies, and it is larger by two orders of magnitude around $10 \mathrm{GeV}$ than the line component.

We checked our calculation by comparison with a similar calculation given by Buch et al ${ }^{[30}$ In Fig. B the spectra of electrons plus positrons from LKP annihilation after propagation are plotted for $m_{B^{(1)}}=1000 \mathrm{GeV}$. Plots indicated by "JB" are given in Ref. ${ }^{30}$ for various combination of parameters: "NFW" or "ISO" (isothermal) for halo density models, "MIN", "MED", and "MAX" for halo propagation models (here "MF1" for the Galactic magnetic field model is assumed). Detailed descriptions of these parameter sets are given in Refs 30 These plots indicate that two halo density models give almost the same spectra, while different halo propagation models affect the spectra especially in the lower energy region. Also shown in Fig. 3 indicated by "MS" is our calculation using the Green function approach based on calucation by Moskalenko and Strong 23 (corresponding to the solid line in Fig. (2). The MS spectrum is larger than JB by a factor of about three just below the LKP mass, however the overall shape in the higher energy region $(\gtrsim 10 \mathrm{GeV})$ of the plots looks similar. We will use only the data in this energy region observed by AMS-02 as discussed below, and those models (MS and JB) give similar results. We also note that the parameters for the Green function used in MS are given for the dark matter mass up to $1000 \mathrm{GeV}$, but we would like to discuss the behavior of the boost factor when the dark matter mass is heavier than $1000 \mathrm{GeV}$. Thus, in the following discussion, we will calculate the electron plus positron spectrum and the positron fraction to set constraints on the boost factor assuming the propagation models of JB only to avoid complicate discussion and readers' confusion.

\section{Discussion}

So far, we have calculated the electron plus positron spectrum from LKP annihilation, which we denote as $F_{\mathrm{LKP}}(E)$. Now, we compare the result of calculation with recent measurements to discuss possible constraints on the boost factor.

Yuan and Bi calculated the cosmic-ray secondary (hereafter "conventional") electron plus positron spectrum, 29 which we denote as $F_{\text {Conv }}(E)$. We note that a normalization factor, $c_{e^{+}}$, is included in their calculation to fit the observational data, so we treat that the overall normalization factor of $F_{\text {Conv }}(E)$ as a free parameter, which reduces a degree of freedom by unity.

Then, we assume the total electron plus positron spectrum as follows

$$
\Phi^{e^{ \pm}}(E)=B_{f} \times F_{\mathrm{LKP}}(E)+C \times F_{\mathrm{Conv}}(E)
$$

where a coefficient $C$ is a normalization factor, which corresponds to $c_{e^{+}}$in Ref ${ }^{29}$ Here we employ the least-squares method to obtain appropriate values for $B_{f}$ and 


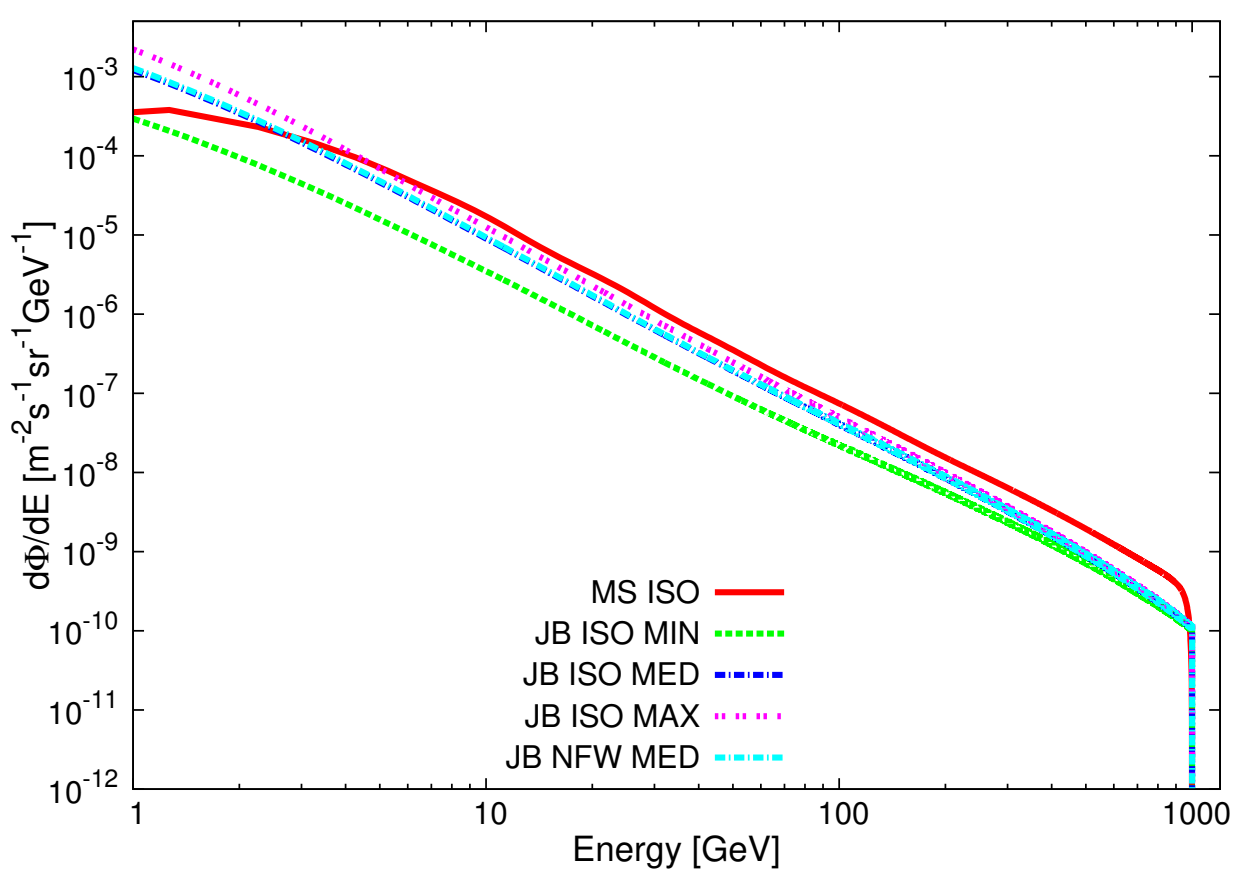

Fig. 3. (Color Online). The spectra of electrons plus positrons from LKP annihilation after propagation for $m_{B(1)}=1000 \mathrm{GeV}$. The solid line corresponds to solid line in Fig. 22 based on calculation given by Moskalenko and Strong, "MS". Other lines show the spectra based on calculation given by J. Buch, "JB", for each halo density and propagation model (see text). We assume the boost factor $B_{f}=1$.

$C$ to fit the AMS-02 data! $\frac{15}{15}$ The goodness of the fit can be tested by the sum

$$
\chi^{2}=\sum_{i} \frac{(\text { data }- \text { model })^{2}}{\sigma_{\text {data }}^{2}}
$$

where "data" means the data points, "model" is given by Eq. (5), and we assume $\sigma_{\text {data }}=\sigma_{\text {stat }}+\sigma_{\text {syst }}$ as a conservative estimate of the error of the data points as the worst case, instead of $\sigma_{\text {data }}=\sqrt{\sigma_{\text {stat }}^{2}+\sigma_{\text {syst }}^{2}}$ as is usually assumed. Here, $\sigma_{\text {stat }}$ and $\sigma_{\text {syst }}$ are the statistic and systematic errors for the electron plus positron spectrum quoted by the AMS-02 collaboration! $\frac{[15}{15}$ The index $i$ runs the data points in the energy range between about 30 and $1000 \mathrm{GeV}$. A number of degrees of freedom is 29 (= a number of data points (31) minus unknown parameter (2)). Thus, $\chi^{2}<49.6$ is required to be consistent with the AMS-02 data at $99 \%$ confidence level.

We calculate $\chi^{2}$ values for various parameter sets, where we vary the factor $C$ from 1 to 2 in 0.1 step and the boost factor $B_{f}$ from 0 to 1500 in 5 step. The results of this calculation show that we have no parameter set to fit AMS-02 data at $99 \%$ confidence level unless the factor $C=1.2$, even if we take any value for the boost factor (from 0 to 1500). When we set $C$ equals to 1.2 , we can obtain a range of the 
Table 1. The values of the boost factor assuming Isothermal (NFW) halo model and for each propagation model with the best fit (B), lower (L) and upper (U) limit to AMS-02 obeservational spectrum.

\begin{tabular}{c|ccc|ccc|ccc}
\hline $\begin{array}{c}\text { LKP mass } \\
{[\mathrm{GeV}]}\end{array}$ & \multicolumn{4}{|c|}{ MIN } & \multicolumn{4}{|c|}{ MED } & \multicolumn{3}{c}{ MAX } \\
\hline 500 & $0(0)$ & $35(35)$ & $125(125)$ & $0(0)$ & $20(20)$ & $80(80)$ & $0(0)$ & $20(20)$ & $70(65)$ \\
1000 & $0(0)$ & $105(105)$ & $390(390)$ & $0(0)$ & $70(65)$ & $240(240)$ & $0(0)$ & $60(55)$ & $205(200)$ \\
1200 & $0(0)$ & $145(145)$ & $530(535)$ & $0(0)$ & $90(90)$ & $325(320)$ & $0(0)$ & $80(75)$ & $275(265)$ \\
1500 & $0(0)$ & $210(210)$ & $775(775)$ & $0(0)$ & $130(130)$ & $465(460)$ & $0(0)$ & $110(105)$ & $390(375)$ \\
\hline \hline
\end{tabular}

values of $B_{f}$ under the condition $\chi^{2}<49.6$ for each LKP mass, halo density, and propagation model, which are given in Table 1, The result for the case of $B_{f}=0$ implies that we can explain the total electron plus positron spectrum observed by AMS-02 without LKP contribution. The resulting total electron plus positron

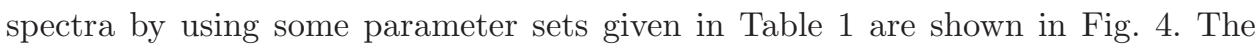
dot-dashed line shows the spectrum with the best fit boost factor, $B_{f}=130$, for isothermal halo model and MED propagation model for $m_{B^{(1)}}=1500 \mathrm{GeV}$. Thick solid lines are the spectral fits to the AMS-02 data with upper-limit boost factors, where $B_{f}=80,240,465$ for $m_{B^{(1)}}=500,1000,1500 \mathrm{GeV}$, respectively. Thin dashed line shows a conventional spectrum $\frac{29}{29}$ multiplied by $C=1.2$. This figure shows the edge structure will be clearer for heavier LKP mass, and we can expect to differentiate such structures in the higher energy region by future observations with higher sensitivity.

Now, we discuss the constraints on the boost factor $B_{f}$ based on the positron fraction observed by AMS- 02.5 The positron fraction, $e^{+} /\left(e^{-}+e^{+}\right)$, for the conventional spectrum, $f_{\text {Conv }}(E)$, depends on energy but is generally smaller than 0.1 .29 On the other hand, the LKP pair annihilation creates the same number of electrons and positrons, so the positron fraction for the LKP spectrum, $f_{\mathrm{LKP}}$, always equals to 0.5 . Then, the total positron fraction is given by

$$
\text { Positron Fraction }=\frac{F_{\mathrm{LKP}}(E) \times B_{f} \times f_{\mathrm{LKP}}+F_{\mathrm{Conv}}(E) \times f_{\mathrm{Conv}}(E)}{F_{\mathrm{LKP}}(E) \times B_{f}+F_{\mathrm{Conv}}(E)},
$$

Then, we employ the least-squares method to obtain the appropriate value for $B_{f}$ to fit the AMS-02 data. ${ }^{5}$ In a similar way with a analysis of the total electron plus positron spectrum, the goodness of the fit can be tested by the sum as Eq. (6). In this case, "model" is given by Eq. (77), and the data points "data", $\sigma_{\text {stat }}$ and $\sigma_{\text {syst }}$ are the statistic and systematic errors for positron fraction quoted by the AMS-02 collaboration. ${ }^{5}$ The index $i$ runs the data points in the energy range between 10 and about $400 \mathrm{GeV}$. A number of degrees of freedom is $42(=43-1)$. Thus, $\chi^{2}<66.2$ is required to be consistent with the AMS-02 data at $99 \%$ confidence level. We obtain a range of the values of $B_{f}$ under the condition $\chi^{2}<66.2$ for each LKP mass, halo density and propagation models. With this prescription, we calculate the positron fractions for several assumed LKP masses and propagation models as a function of energy to fit the AMS-02 dat $\mathrm{5}^{5}$ as shown in Fig. 5. One can see the observed data can be fit well by adding the LKP flux with an appropriate boost factor, and the 


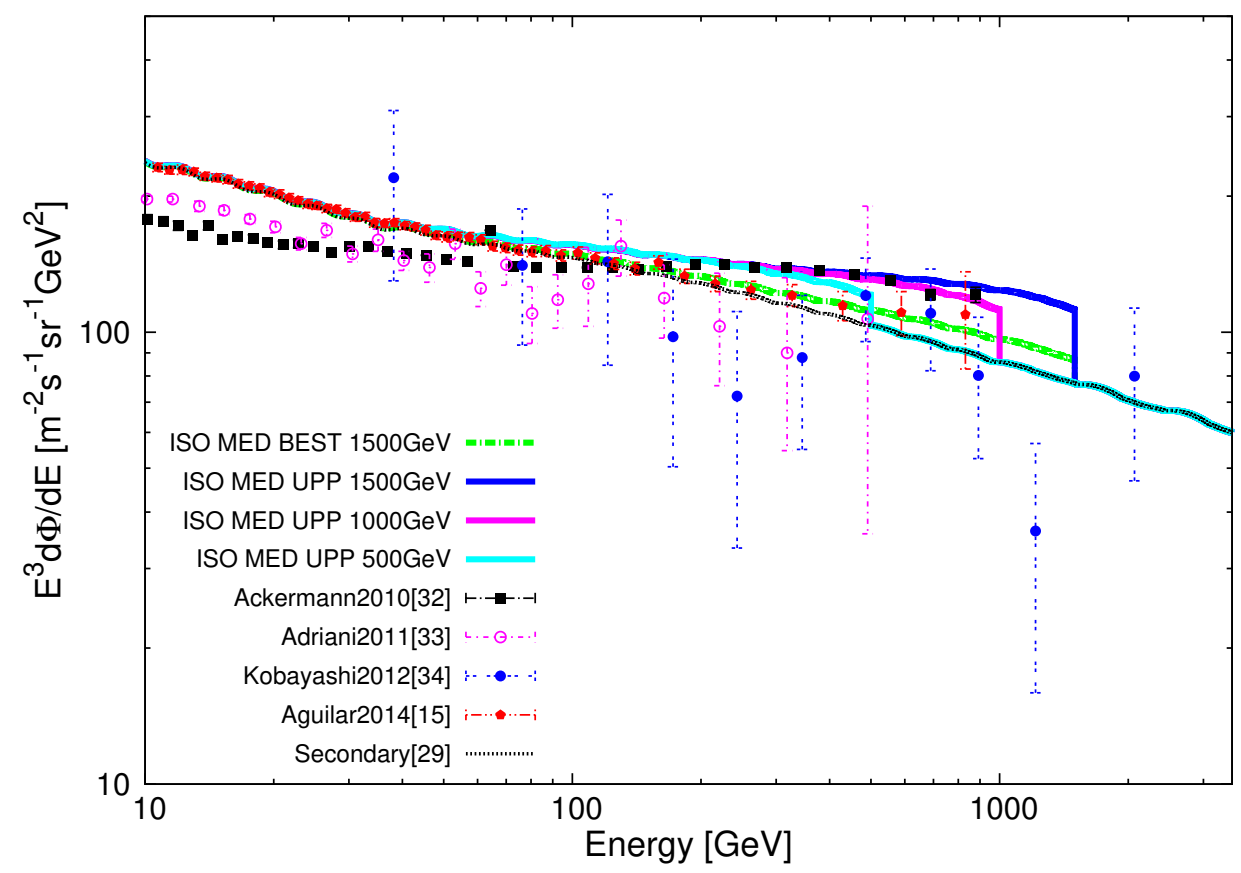

Fig. 4. (Color Online). The electron plus positron spectra assuming several LKP masses, $m_{B^{(1)}}$, and boost factors, $B_{f}$. The dot-dashed line shows the spectrum fit to AMS-02 data with the best fit boost factor, $B_{f}=130$, for isothermal halo model and MED propagation model for $m_{B^{(1)}}=1500 \mathrm{GeV}$. Thick solid lines are the spectral fits to the AMS-02 data with upper-limit boost factor, $B_{f}=80,240,465$ for $m_{B^{(1)}}=500,1000,1500 \mathrm{GeV}$, respectively. Also shown are the recent observational data, $\frac{15234}{32}$ and an adjusted (factor $C=1.2$ is multiplied) prediction spectrum by the cosmic-ray secondary calculation (thin dotted line)!29

Table 2. The value of boost factor assuming Isothermal (NFW) halo model and each propagation model with the best fit (B), lower (L) and upper (U) limit to AMS02 obeservational data (Positron Fraction).

\begin{tabular}{|c|c|c|c|c|c|c|c|c|c|}
\hline $\begin{array}{l}\text { LKP mass } \\
{[\mathrm{GeV}]}\end{array}$ & $B_{f} \mathrm{~L}$ & $\begin{array}{l}\text { MIN } \\
B_{f} \mathrm{~B}\end{array}$ & $B_{f} \mathrm{U}$ & $B_{f} \mathrm{~L}$ & $\begin{array}{l}\text { MED } \\
B_{f} \mathrm{~B}\end{array}$ & $B_{f} \mathrm{U}$ & $B_{f} \mathrm{~L}$ & $\begin{array}{l}\text { MAX } \\
B_{f} \mathrm{~B}\end{array}$ & $B_{f} \mathrm{U}$ \\
\hline 500 & $343(343)$ & $393(393)$ & $444(444)$ & $152(149)$ & $178(173)$ & $203(199)$ & $116(101)$ & $135(118)$ & $154(135)$ \\
\hline 1000 & $983(984)$ & 1150 (1150) & $1320(1320)$ & $467(456)$ & $519(506)$ & $571(555)$ & 353 (308) & 390 (341) & $428(375)$ \\
\hline 1200 & 1286 (1287) & $1500(1500)$ & 1717 (1716) & $627(612)$ & $679(661)$ & $732(710)$ & $473(415)$ & $510(446)$ & $547(477)$ \\
\hline 1500 & 1775 (1775) & $2058(2058)$ & 2199 (2199) & $909(894)$ & 937 (913) & 964 (931) & No Fit & No Fit & No Fit \\
\hline
\end{tabular}

energy dependence of the data can be fit better if we include not only the "line" but also the "continuum" component. Also for that case using the MED propagation model seems to give a better fit. The values of the boost factor, $B_{f}$, derived as above for each LKP mass are given in Table 2. If we assume LKP mass $=1500 \mathrm{GeV}$ and MAX propagation model, there would be no allowed value for $B_{f}$ to be consistent with the positron fraction observed by AMS-02 at $99 \%$ confidence level.

From the results given in Tables 1 and 2, we observe the lower limit on $B_{f}$ to 


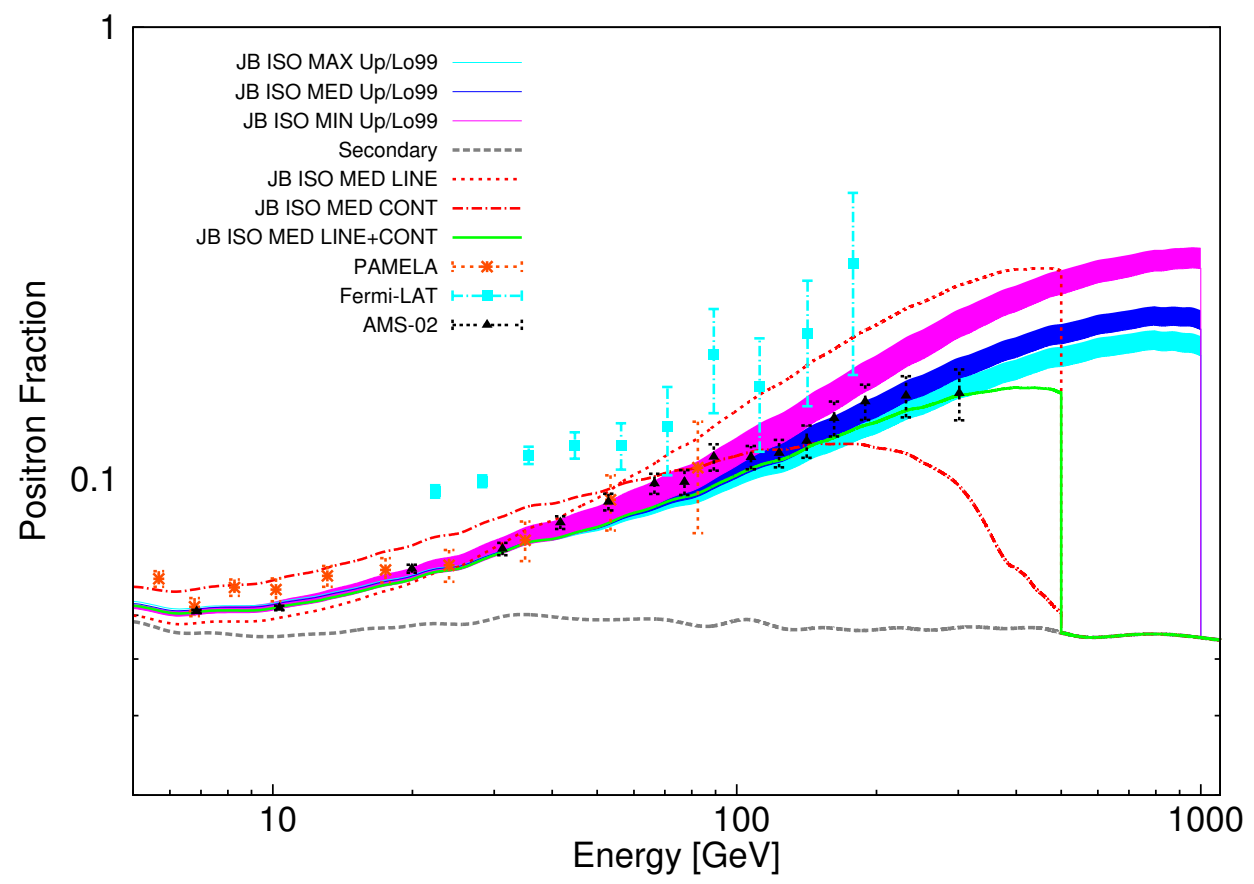

Fig. 5. (Color Online). The positron fraction expected from LKP annihilation for each propagation model compared with recent measurements, $\frac{3}{5}$ and a prediction by the cosmic-ray secondary electron and positron calculation (thin dashed line) $\frac{29}{29}$ Thick solid lines show the range of expected positron fraction by using obtained upper and lower limit on $B_{f}$ at $99 \%$ confidence level for each propagation model assuming $m_{B^{(1)}}=1000 \mathrm{GeV}$. Thin solid line shows the expected positron fraction including not only the line component but also the continuum component, thin dotted line shows that without the continuum component, and thin dot-dashed line shows that without the line component for MED propagation model and $m_{B^{(1)}}=500 \mathrm{GeV}$.

be consistent with the positron fraction may be larger than the upper limit on $B_{f}$ to be consistent with the electron plus positron spectrum. Although we can fit to the positron fraction and the electron plus positron spectrum separately by adding LKP contribution, the required boost factors differ significantly and it is difficult to explain both the electron plus positron spectrum and the positron fraction at the same time, if we only take account of observational data obtained by AMS-02. However, there remains rather large differences in the total electron plus positron spectrum among various experiments, so it is a bit too early to conclude whether LKP survives as a relevant dark matter candidate or not.

It is important to compare our results with those given in previous studies. The detectability of gamma rays from dark matter annihilation has been discussed in many literatures. For instance, Bergström et al ${ }^{\sqrt[35]{5}}$ discussed the relation between the mass of dark matter and cross section, and predicted an upper limit of the cross section about $9 \times 10^{-28} \mathrm{~cm}^{3} \mathrm{~s}^{-1}$ assuming $1000 \mathrm{GeV}$ dark matter mass for HESS- 
II observation. Assuming $130 \times 10^{-6} \mathrm{pb}$ for the cross section for annihilation into photon pairs, this upper limit corresponds to about $B_{f}=230$. In our calculation, by assuming $m_{B^{(1)}}=1000 \mathrm{GeV}$, Isothermal halo model and MED halo propagation based on the JB propagation, we obtain $B_{f}=0-240$ (to explain the total spectrum) or 467-571 (to explain the positron fraction), which fit to AMS-02 observational data at $99 \%$ confidence level. Thus, the upper limit imposed by gamma-ray observation is comparable with our result. In addition, $B_{f}$ is also calculated in the

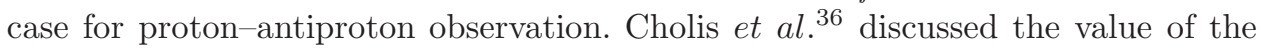
boost factor based on the data of proton-antiproton flux obtained by the PAMELA observation. They pointed out the value of $B_{f}$ should be $\mathcal{O}(100)$ for $1000 \mathrm{GeV}$ dark matter mass, which is not contradiction with our result.

In the energy region around or higher than $1000 \mathrm{GeV}$, the measurements suffer rather large statistical uncertainties. Thus, we hope on-going (CALET ${ }^{37}$ and DAMPE ${ }^{38}$ ) and near-future missions with better sensitivity will clarify the existence (or non-existence) of the LKP dark matter.

\section{Conclusion}

We investigated the cosmic electron and positron spectra from LKP annihilation taking account of propagation effects in the Galaxy. We paid particular attention to the calculation of the "continuum" emission, which is a secondary product of LKP annihilation, in addition to the "line" component directly produced by annihilation. The result shown in Fig. 2 indicates the "continuum" component dominates over the "line" component in the low energy region after propagation in the Galactic halo to Earth, and changes the shape of the positron fraction, as shown in Fig. 5 We also consider the spectra for different halo density and propagation models. The results mean that the difference of halo density models do not affect on the spectra effectively, but the choice of halo propagation models change the shape of spectra in the lower energy region, as shown in Fig. 3 .

We estimated the value of the boost factor to enhance the halo density in the Galactic center region by using the electron plus positron spectrum and the positron fraction measurement by AMS-02, which is given in Tables 1 and 2 The results of our calculation imply that while the addition of the LKP component gives good fits to the total electron plus positron spectrum and the positron fraction with appropriate boost factors estimated for each case, but these two boost factors are not consistent each other, if we only take account of the AMS-02 observation. However, considering the rather large experimental uncertainties we should not conclude that whether LKP can be a good candidate of cold dark matter now.

If the characteristic structure in electron plus positron flux is observed in near future, we may conclude dark matter is made of LKP. It would be a conclusive evidence for the existence of extra dimensions. 


\section{Acknowledgments}

We would like to thank Mr. Akihiko Kawamura and Dr. Fumihiro Matsui for useful discussions and helpful comments. We also would like to thank Marco Cirelli and his collaborators for supplying numerical data of their calculation.

\section{References}

1. See, for example, K. Freese, B. D. Fields, and D. S. Graff, in Proceedings of the MPA/ESO Workshop on the First Stars, Garching, Germany, 1999, arXiv:astro-ph/0002058

2. P. A. R. Ade et al, Astron. Astrophys. 571, A16 (2014).

3. O. Adriani et al., Astropart. Phys. 34, 1 (2010).

4. M. Ackermann et al., Phys. Rev. Lett. 108, 011103 (2012)

5. M. Aguilar et al., Phys. Rev. Lett. 110, 141102 (2013); See also its Supplemental Material;

L. Accardo et al. (AMS Collaboration), Phys. Rev. Lett. 113, 121101 (2014).

6. L. Feng et al., Phys. Lett. B 728, 250 (2014).

7. J. Kopp, Phys. Rev. D 88, 076013 (2013).

8. F. Calore et al., Phys. Rev. D 91, 063003 (2015).

9. T. Appelquist, H. C. Cheng, and B. A. Dobrescu, Phys. Rev. D 64, 035002 (2001).

10. H. C. Cheng, K. T. Matchev, and M. Schmaltz, Phys. Rev. D 66, 036005 (2002).

11. G. Servant and T. M. P. Tait, Nucl. Phys. B650, 391 (2003).

12. H. C. Cheng, J. L. Feng, and K. T. Matchev, Phys. Rev. Lett. 89, 211301 (2002).

13. A. A. Moiseev et al., Frascati Physics Series VVV, 1 (2007).

14. S. Ting, Talk at the 33rd Int. Cosmic Ray Conference, Rio de Janeiro, 2-9 July 2013 (2013).

15. M. Aguilar et al. (AMS Collaboration), Phys. Rev. Lett. 113, 221102 (2014).

16. S. Torii for the CALET collaboration, Proc. 34th International Cosmic Ray Conference (ICRC2015), 0581, Proc of Science, in press.

17. L. Bergström, T. Bringmann, M. Eriksson, and M. Gustafsson, Phys. Rev. Lett. 94, 131301 (2005).

18. G. Bélanger et al., J. Cosmol. Astropart. Phys. 02, 009 (2011).

19. G. Aad et al. (The ATLAS collaboration), JHEP 04, (2015) 116.

20. D. Hooper and G. D. Kribs, Phys. Rev. D 67, 055003 (2003).

21. M. Cirelli et al., J. Cosmol. Astropart. Phys. 1103 (2011) 051. Erratum: J. Cosmol. Astropart. Phys. 1210 (2012) E01.

22. P. Ciafaloni et al., J. Cosmol. Astropart. Phys. 1103 (2011) 019.

23. I. V. Moskalenko and A. W. Strong, Phys. Rev. D 60, 063003 (1999).

24. F. Prada et al., Phys. Rev. Lett. 93, 241301 (2004).

25. J. F. Navarro, C.S. Frenk, and S. D. White, Astrophys. J. 462, 563 (1996).

26. L. Bergström, New J. Phys. 11, 105006 (2009).

27. M. Cirelli et al., Nucl. Phys. B 813, 1 (2009); B873, 530 (2013) [addendum].

28. T. Stanev, "High Energy Cosmic Rays", Springer Verlag (2003).

29. Q. Yuan and X. J. Bi, Phys. Lett. B727, 1 (2013).

30. J. Buch et al., J. Cosmol. Astropart. Phys. 09, 037 (2015).

31. F. Donato et al., Phys. Rev. D 69, 063501 (2004).

32. M. Ackermann et al., Phys. Rev. D 82, 092004 (2010).

33. O. Adriani et al., Phys. Rev. Lett. 106, 201101 (2011).

34. T. Kobayashi et al., Astrophys. J 760, 146 (2012). 
February 28, $2022 \quad$ 22:26 WSPC/INSTRUCTION FILE ele“ijmpd’arxiv

The electron plus positron spectrum from annihilation of Kaluza-Klein dark matter in the Galaxy

35. L. Bergström et al., J. Cosmol. Astropart. Phys. 11, 025 (2012).

36. I. Cholis J. Cosmol. Astropart. Phys. 09, 007 (2011).

37. S. Torii, Nucl. Instrum. Methods. A630, 55 (2011).

38. X. Wu, Proc. 34th International Cosmic Ray Conference (ICRC2015), 1192, Proc of Science, in press. 